\title{
La influencia de la personalidad en los estilos de liderazgo
}

\section{The influence of personality on leadership styles}

Méndez Bravo, J. C.

Universidad de Guayaquil, Ecuador

Méndez Bravo, M. A.

Universidad de Guayaquil, Ecuador

Autor por Correspondencia: ing.com.manuelmendez@ hotmail.com, julio.mendezb@ug.edu.ec Fecha de recepción: 12 de Septiembre de 2016 - Fecha de aceptación: 01 de Noviembre de 2016

Resumen: El presente trabajo plantea determinar si el tipo de liderazgo desarrollado por una persona para dirigir una organización o un grupo de trabajo, está determinado por la personalidad del individuo y de cómo se puede adaptar con las personalidades de sus subalternos para desarrollar actividades organizadas y direccionadas al éxito y cumplimiento de los objetivos planteados. Para lo cual se ha realizado una revisión literaria de diferentes investigaciones contraponiendo resultados y desde una perspectiva cualitativa determinar si el liderazgo de un líder está definido por la personalidad. Las personas que tienen tipos de personalidad orientados a tareas tienden a tener una considerable atención en los detalles. Ellos no se sienten cómodos de iniciar una acción hasta que están satisfechos que tienen todos los elementos necesarios. Por otro lado, las personas que tienen relaciones orientado a los tipos de personalidad tienden a tener un interés considerable en el resultado y se sienten cómodos de iniciar una acción de planta cuando tienen sólo los hechos esenciales (Blake \& Mouton, 1982). Por lo tanto, es importante para un líder entender la personalidad y ajustar con precisión el estilo de liderazgo a la situación de la gestión. (Bass, 1990). Palabras claves: contrato psicológico; compromiso organizacional; liderazgo

Abstract: This paper presents determine whether the type of leadership developed by an individual to lead an organization or a working group, is determined by the personality of the individual and how they can adapt to the personalities of their subordinates to develop organized activities and addressed to success and fulfillment of the objectives. For which you have performed a literature review of various studies contrasting results from a qualitative perspective and determine whether the leadership of a leader is defined by personality. People with personality types task-oriented tend to have considerable attention to detail. They do not feel comfortable to bring an action until they are satisfied they have all the necessary elements. On the other hand, people who have oriented relations personality types tend to have a substantial interest in the outcome and feel comfortable to bring an action plant when they have only the essential facts (Blake \& Mouton, 1982). Therefore, it is important for understanding personality and precisely adjust leadership style to the situation of management leader. (Bass, 1990).

Key words: psychological contract; organizational commitment; leadership 


\section{Introducción}

El liderazgo ha sido definido como "la influencia interpersonal, ejercida en situaciones y dirigida a través del proceso de comunicación, hacia el logro de una meta o metas especificadas" (Meredith y Mantel, 2012). Mientras que los rasgos de personalidad se definen como patrones consistentes de pensamientos, sentimientos o acciones que distinguen a las personas entre sí (Yahaya, N. Aslan, M. Taib, M. Ismail1, J. Shariff, Z. Yahaya, A. Boon, Y \& Hashim, S. 2011).

Dado que el liderazgo es influenciado por el tipo de personalidad, es importante primero analizar los tipos de personalidad que pueden definir el estilo de liderazgo en quienes dirigen una organización o grupo de trabajo. Un modelo de cinco factores de la personalidad (a menudo llamado The Big Five) puede ser usado para describir los aspectos más sobresalientes de la personalidad (Goldberg, 1990)

Las dimensiones que componen el modelo de cinco factores son neuroticismo, extraversión, apertura a la experiencia, agradabilidad, y escrupulosidad. Neuroticismo representa la tendencia a padecer de problemas para el ajuste emocional y la experiencia negativa afecta, como la ansiedad, la inseguridad y la hostilidad. Extraversión representa la tendencia a ser alegre, enérgico, activo, y de experimentar efectos positivos, tales como la energía y el celo. La apertura a la experiencia es la disposición a ser imaginativos, no conforme, no convencional, y autónomo. La Agradabilidad es la tendencia a ser confiado, dócil, el cuidado y suave y la escrupulosidad se compone de dos facetas relacionadas: el rendimiento y la fiabilidad (Judge, Bono, Ilies, \& Gerhardt, 2002)

En contraste con ser percibido como un líder, la efectividad del liderazgo se refiere a la actuación de un líder para influir y orientar las actividades de su unidad hacia el logro de sus objetivos (ver Stogdill, 1950).

Considerando lo antes expuesto y los resultados de la investigación "Personality and Leadership: A Qualitative and Quantitative Review” de Judge, Bono, Ilies, \& Gerhardt, donde concluyen "Los resultados de este meta-análisis muestran que, en general, la extraversión, conciencia, apertura, y la neurosis son rasgos útiles en relación con el liderazgo. En conjunto, los resultados proporcionan apoyo a la relevancia del modelo de cinco factores"

Lo antes citado demuestra como la incidencia de la personalidad tiende a determinar el liderazgo de una persona en una organización o grupo de trabajo, adicional a esto se puede recalcar que los tipos de personalidad pueden ser utilizados como complemento no solo en la selección del líder, sino también en la selección del equipo de trabajo.

A continuación se utilizara información realizada en la investigación: Determinants of Leadership Style in Big Five Personality Dimensions, de quienes desarrollaron una matriz de los estilos de liderazgo, donde: Los estilos de liderazgo (LS1 a LS4) se pueden ver a lo largo de un continuo de enfoque y la toma de decisiones centralizada gradualmente cambiando de líder a los subordinados. LS5 es un caso excepcional y se incluye debido a su importancia para entender un modelo completo de la dirección (Hassan, Asad \& Hoshino, 2016). 


\begin{tabular}{|c|c|c|c|c|c|}
\hline & LS1 & LS2 & LS3 & LS4 & LS5 \\
\hline Rol del Líder & $\begin{array}{c}\text { Definir } \\
\text { claramente las } \\
\text { instrucciones y } \\
\text { normas de } \\
\text { funcionamiento }\end{array}$ & $\begin{array}{l}\text { Fomentar la } \\
\text { participación }\end{array}$ & $\begin{array}{l}\text { Buscar más } \\
\text { altos estándares } \\
\text { de excelencia }\end{array}$ & $\begin{array}{l}\text { Ayudar a los } \\
\text { seguidores }\end{array}$ & $\begin{array}{c}\text { Dejar } \\
\text { seguidores } \\
\text { hacer tarea por } \\
\text { sí mismos }\end{array}$ \\
\hline $\begin{array}{l}\text { Preocupación } \\
\text { del líder para } \\
\text { los demás }\end{array}$ & Poco & Alto & Alto & Muy alto & Poco o nada \\
\hline $\begin{array}{l}\text { distancia con } \\
\text { los seguidores }\end{array}$ & Alto & Moderar & Bajo & Bajo & Alto \\
\hline $\begin{array}{l}\text { La toma de } \\
\text { decisiones de } \\
\text { estilo de líder }\end{array}$ & Unilateral & $\begin{array}{l}\text { Compartida } \\
\text { decisiones a } \\
\text { través de los } \\
\text { seguidores de } \\
\text { decisiones }\end{array}$ & $\begin{array}{l}\text { La toma de } \\
\text { decisiones } \\
\text { compartida por } \\
\text { persuasión }\end{array}$ & $\begin{array}{l}\text { La toma de } \\
\text { decisiones } \\
\text { compartida en el } \\
\text { interés de } \\
\text { seguidores }\end{array}$ & $\begin{array}{l}\text { Mínima o } \\
\text { ninguna } \\
\text { lenguado en la } \\
\text { toma de } \\
\text { decisiones }\end{array}$ \\
\hline $\begin{array}{l}\text { La motivación } \\
\text { de los } \\
\text { seguidores }\end{array}$ & $\begin{array}{l}\text { Seguidores son } \\
\text { incapaces de } \\
\text { realizar tareas por } \\
\text { sí mismos y están } \\
\text { motivados } \\
\text { moderadamente }\end{array}$ & $\begin{array}{c}\text { Seguidores } \\
\text { son iguales con } \\
\text { el líder y } \\
\text { altamente } \\
\text { motivado }\end{array}$ & $\begin{array}{c}\text { Identificar } \\
\text { seguidores con } \\
\text { los líderes y } \\
\text { altamente } \\
\text { motivados }\end{array}$ & $\begin{array}{c}\text { Seguidores } \\
\text { tratan de llegar a } \\
\text { su nivel de } \\
\text { autorrealización }\end{array}$ & $\begin{array}{l}\text { Seguidores } \\
\text { se sienten } \\
\text { frustrados y } \\
\text { desmotivados }\end{array}$ \\
\hline $\begin{array}{l}\text { Centrarse en } \\
\text { crecimiento de } \\
\text { los seguidores }\end{array}$ & $\begin{array}{c}\text { Ninguno como } \\
\text { líder enfatiza solo } \\
\text { en los seguidores } \\
\text { para seguir } \\
\text { instrucciones }\end{array}$ & $\begin{array}{c}\text { Moderada } \\
\text { como líder } \\
\text { proporciona } \\
\text { formación y } \\
\text { desarrollo a los } \\
\text { seguidores }\end{array}$ & $\begin{array}{l}\text { Moderada alta } \\
\text { como el } \\
\text { enfoque líder en } \\
\text { el desarrollo de } \\
\text { las } \\
\text { competencias } \\
\text { de los } \\
\text { seguidores }\end{array}$ & $\begin{array}{c}\text { Alta como la } \\
\text { máxima prioridad } \\
\text { del líder es ayudar } \\
\text { a otros a alcanzar } \\
\text { sus objetivos }\end{array}$ & $\begin{array}{l}\text { Ninguno } \\
\text { como líder } \\
\text { sigue sin } \\
\text { involucrarse }\end{array}$ \\
\hline
\end{tabular}

Figura 1: Representación de estilos de liderazgo

Elaborado por: Hassan, Asad \& Hoshino, (2016).

Como se puede observar en la matriz de los estilos de liderazgo donde se puede observar las características que tienen cada estilo de liderazgo y que claramente son marcados por las diferentes personalidades de los individuos que guían las organizaciones.

La personalidad de cada individuo es propia y exclusivas de cada persona, sin embargo la personalidad puede ser determinada por diferentes factores y entornos, lo que a su vez definirá el estilo de liderazgo que se desee aplicar en la organización o grupo de trabajo.

Las personas que tienen diferentes orígenes tienen diferentes actitudes, valores y normas. Estas personas no reflejan su herencia cultural, que son, de hecho, diferente. Estas diferencias dan lugar a diferentes personalidades de los individuos que determinan sus acciones y comportamientos. Algunas personas tienen personalidades fuertes. Pueden influir en otros para actuar y hacer cosas. Otros, que tienen cierto tipo de personalidad, pueden determinar la forma en que las organizaciones se comportan. De hecho, muchos investigadores han llevado a cabo estudios con el fin de entender la relación entre la personalidad y comportamientos humanos. (Dole y Schroeder, 2001). 
Dado que la personalidad determina el comportamiento de la organización como se manifiesta en la cita previa, esta información soporta la tesis que la personalidad influye el tipo de liderazgo y como consecuencia la forma que se desarrollara.

Los líderes se enfrentan a un trabajo muy duro para mantener a la gente enfocada y motivada hacia el logro de los objetivos, los cuales están destinados a ser logrado. Los líderes que necesitan las organizaciones deben ser aquellos que pueden guiar a las personas a través de la incertidumbre y la confusión, que los períodos de cambio rápido conllevan (Hussein, A. AbuJarad, I. Sulaiman, M. \& Nikbin, D. 2011)

Entendiendo que cada organización también buscara líderes con estilos definidos según su personalidad, y acorde al momento o estado de la organización.

Los estilos de liderazgo en las organizaciones también se han encontrado para tener un profundo impacto en los resultados organizativos y subalternos (Boerner, Eisenbeiss, \& Griesser, 2007; Felfe \& Schyns, 2004; Hater \& Bass, 1988; Howell \& Avolio, 1993; Ismail, Baizura, \& Rabaah, 2009; Zhu, Chew, \& Spangler., 2005). Esta información ratifica lo afirmado al inicio del trabajo desarrollado.

Los líderes también capaz de mover, motivar e inspirar a su equipo y las partes interesadas a aceptar el cambio y las estrategias, tácticas y tareas necesarias para lograr las metas que nos ocupa (Prabhakar, 2005).

Los líderes desempeñan un papel clave en la construcción de equipos eficaces, la creación de un marco para dirigir sus actividades y motivarlos a mantener la concentración. La formación de equipos es una actividad permanente que es una de las principales responsabilidades del administrador y debe ser incorporado en las actividades del día a día. El líder es el eslabón fundamental para la misión y objetivos de la organización y el trabajo en equipo de primera línea (Galvin, T. Gibbs, M. \& Williams, C. 2014).

Hoy en día las organizaciones están en busca de líderes capaces de utilizar y aprovechar al máximo todos sus recursos disponibles, sino también que brinde a la organización la posibilidad para poder compartir y retener el conocimiento desarrollado por los miembros de la organización para con esto contribuir a la sostenibilidad de la organización en el tiempo.

Los líderes aplican diferentes estrategias en situaciones de gestión de la emoción líder y el seguidor (Diefendorff \& Yang, 2008). Lo que significa que el líder debe tener una personalidad que sobresalga de los demás miembros de la organización y que pueda influir de forma positiva para el fiel cumplimiento de los objetivos de la organización.

Williams (1997) ha sugerido que una variedad de rasgos de personalidad son susceptibles de ser asociados con las habilidades de auto-liderazgo de manera significativa. En particular, Williams (1997) propuso asociaciones positivas entre las habilidades de auto-liderazgo y extroversión, estabilidad emocional, la conciencia, la auto-eficacia en general, locus de control interno, y el autocontrol. 
Con la información anterior establecemos que la relación entre la personalidad y el liderazgo es real y vinculante, lo que explica como la personalidad influye directamente en el estilo de liderazgo, el cual va desde un carácter estricto y rígido hasta uno permisivo y que da libertad de decisión a los subalternos.

La relación entre la personalidad y el estilo de liderazgo está confirmado y basado en muchas investigaciones realizadas por muchos autores, en investigaciones de tipo cualitativa así como de tipo cuantitativa, lo que fundamenta claramente el trabajo presentado.

Yukl (citado por (Hussein, A. Abu-Jarad, I. Sulaiman, M. \& Nikbin, D. 2011) define el liderazgo como "el proceso de influir en otros para entender y estar de acuerdo sobre lo que hay que hacer y cómo hacerlo, y el proceso de facilitar los esfuerzos individuales y colectivos para lograr objetivos comunes." La simplificación de la definición, el liderazgo es la capacidad de influir en un grupo hacia el logro de metas.

La personalidad tiene un impacto en el estilo de liderazgo de los líderes y, por lo tanto, deben ser considerados al examinar la efectividad del liderazgo. Sin embargo, el impacto exacto de la personalidad necesita ser examinado más a fondo (Hussein, A. Abu-Jarad, I. Sulaiman, M. \& Nikbin, D. 2011). Esta parte del análisis del trabajo realizado por los investigadores antes mencionados permite relacionar claramente y evidenciar la relación existente entre la personalidad y su influencia en el estilo de liderazgo del individuo que guía la organización y esta encargado de su correcto funcionamiento y cumplimiento de objetivos.

A continuación una tabla donde se establecen las características que tienen los lideres según el estilo de liderazgo que empleen.

\begin{tabular}{|c|c|c|c|c|}
\hline LS1 & LS2 & LS3 & LS4 & LS5 \\
\hline Autocrático & Participativo & Transformacional & Servidor & laissez-faire \\
\hline Transaccional & Interpersonal & Visionario & Ciudadano & \\
\hline Tarea-orientada & Entrenamiento & Carismático & Autentico & \\
\hline Directivo & Afiliativo & Orientado a logros & Orientador humano & \\
\hline Autoritario & Ароуо & Ajuste de pasos & Ético & \\
\hline Aversivo & Relación-orientada & Empoderamiento & & \\
\hline Narcisismo & Autoritario & E-leadership & & \\
\hline Instrumental & Consultor-consejero & Distributivo & & \\
\hline Coercido & Democrático & Intelectual & & \\
\hline \multirow[t]{2}{*}{ Auto protector } & Expresivo & Opinión & & \\
\hline & Orientador de equipo & Autónomo & & \\
\hline & Delegativo & & & \\
\hline LS1-LS5 indican & stilo de liderazgo 1-5 & & & \\
\hline
\end{tabular}

Figura 2: estilos basados en características comunes.

Elaborado por: Hassan, Asad \& Hoshino, (2016)

Como se puede observar las características dentro de cada estilo de liderazgo, son las que definen la personalidad de los líderes de las organizaciones o grupos de trabajo, esta matriz de 
características está relacionada con la figura 1 y en conjunto pueden determinar y soportar la tesis afirmada al inicio.

\section{Metodología}

La metodología cualitativa es una forma sistemática de investigar encaminada a obtener una comprensión más profunda del contexto de experiencias, procesos y comportamientos. Esta metodología proporciona un marco para la investigación centrado en el entendimiento del "por qué" y "cómo" respecto a los comportamientos humanos y creencias, y con frecuencia se utiliza para generar hipótesis o construir teorías (López \& Fuentes, 2013).

La investigación cualitativa no intenta medir la extensión de los fenómenos, sino que busca describir qué existe, cómo varía en las diferentes circunstancias y cuáles son las causas subyacentes. La investigación cualitativa se enfoca en la búsqueda de explicaciones, percepciones, sentimientos y opiniones de los sujetos en estudio. El investigador cualitativo adquiere un punto de vista desde dentro del fenómeno en estudio, aunque mantiene una perspectiva analítica como observador externo. Las técnicas de investigación cualitativas son flexibles, de acuerdo con los requerimientos de la situación prevaleciente, con una extracción del significado de los datos, sin reducirlos a números y sin un análisis estadístico (López \& Fuentes, 2013).

Como indican los autores antes citados, en el presente artículo se busca diferentes perspectivas encontradas en diferentes papers de journals reconocidos y que han sido referenciados en otros trabajos donde se aborda este tema, para ser analizadas y determinar desde la perspectiva de los autores del presente artículo determinar si el liderazgo de un líder está definido por la personalidad.

\section{Conclusión}

Como se puede verificar, después de haber realizado una investigación literaria utilizando papers de journals con investigaciones de tipo cualitativo y cuantitativo donde se realizaron análisis desde diferentes enfoques y con resultados diferentes, se realizó un análisis que concluyo con que existe una relación directa en la influencia del tipo de liderazgo basados en la personalidad del líder de la organización o jefe del grupo de trabajo, sin embargo, es muy importante recalcar que para que el líder pueda realizar una buena gestión y cumpla con fechas y metas de la organización, es necesario que la personalidad de los seguidores o subalternos de quienes forman parte de la organización o grupo de trabajo, se pueda acoplar al estilo de liderazgo y personalidad del líder, ya que personalidades no muy afines pueden generar conflicto

y un ambiente de trabajo toxico que pude afectar a los demás miembros de la organización, razón por la cual se sugiere realizar investigaciones referente al impacto de la personalidad en la interacción del líder con los subalternos, así como analizar variables diferentes como establecer si el pensamiento crítico y el pensamiento estratégico influyen en el estilo del liderazgo de un lider.

\section{Bibliografía}


Diefendorff, J. M., Richard, E. M., \& Yang, J. (2008). Linking emotion regulation strategies to affective events and negative emotions at work. Journal of Vocational Behavior, 73, 498508.

Dole, C. \& Schroeder, R. (2001) "The impact of various factors on the personality, job satisfaction and turnover intentions of professional accountants", Managerial Auditing Journal. 16 (4), p. $(234-245)$

Galvin, T. Gibbs, M. \& Williams, C. (2014). Leadership Competencies of Project Managers: An Empirical Study of Emotional, Intellectual, and Managerial Dimensions. Journal of Economic Development, Management, IT, Finance and Marketing. 6(1). p (35-60).

Goldberg, L. R. (1990). An alternative "description of personality": The Big-Five factor structure. Journal of Personality and Social Psychology. 59. p (1216-1229)

Hassan, Asad \& Hoshino, (2016). Determinants of Leadership Style in Big Five Personality Dimensions. Universal Journal of Management. 4(4): 161-179

Hussein, A. Abu-Jarad, I. Sulaiman, M. \& Nikbin, D. (2011). The Impact of Personality and Leadership Styles on Leading Change Capability of Malaysian Managers. Australian Journal of Business and Management Research. 1(2). p (70-99).

Judge, T. Bono, J. Ilies, R. \& Gerhardt, M. (2002). Personality and Leadership: A Qualitative and Quantitative Review. Journal of Applied Psychology. Vol. 87, No. 4, 765-780

López, E., \& Fuentes, (2013). Metodología cualitativa: un cambio de paradigma en la investigación médica, Revista de Sanidad Militar. 67(4), p. 161-164.

Noordin Yahaya, Mohammad Aslan B, Mohammad Taib, Jasmi Ismail1, Zainudin Shariff, Azizi Yahaya, Yusof Boon \& Sharin Hashim. (2011). African Journal of Business Management. Vol. 5(22), pp. 9635-9648

Prabhakar, G. (2005). Switch leadership in projects: An empirical study reflecting the importance of transformational leadership on project success across twenty-eight nations. Project Management Journal, 36(4), 53-60.

Williams, S. (1997). Personality and self-leadership. Human Resource Management Review. 7. p $(139-155)$ 